\title{
Search for manifestations of new physics in fermion-pair production at LEP
}

\section{L3 Collaboration}

M. Acciarri ${ }^{\mathrm{z}}$, P. Achard $^{\mathrm{s}}$, O. Adriani ${ }^{\mathrm{p}}, \mathrm{M}$. Aguilar-Benitez ${ }^{\mathrm{y}}, \mathrm{J}$. Alcaraz $^{\mathrm{y}}$, G. Alemanni ${ }^{\mathrm{v}}$, J. Allaby ${ }^{\mathrm{q}}$, A. Aloisio ${ }^{\mathrm{ab}}$, M.G. Alviggi ${ }^{\mathrm{ab}}$, G. Ambrosi ${ }^{\mathrm{s}}$, H. Anderhub av , V.P. Andreev f,aj, T. Angelescu ${ }^{1}$, F. Anselmo ${ }^{\mathrm{i}}$, A. Arefiev ${ }^{\text {aa }}$, T. Azemoon ${ }^{\text {c }}$, T. Aziz ${ }^{\text {j }}$, P. Bagnaia ${ }^{\text {ai }}$, A. Bajo ${ }^{\mathrm{y}}$, L. Baksay $^{\mathrm{aq}}$, A. Balandras ${ }^{\mathrm{d}}$, S.V. Baldew ${ }^{\mathrm{b}}$, S. Banerjee ${ }^{\mathrm{j}}$, Sw. Banerjee ${ }^{\mathrm{j}}$, A. Barczyk ${ }^{\text {av,at }}$, R. Barillère ${ }^{\mathrm{q}}$, L. Barone $^{\text {ai }}$, P. Bartalini ${ }^{\mathrm{v}}$, M. Basile ${ }^{\mathrm{i}}$, R. Battiston ${ }^{\text {af }}$, A. Bay ${ }^{\mathrm{v}}$, F. Becattini ${ }^{\mathrm{p}}$, U. Becker ${ }^{n}$, F. Behner ${ }^{\text {av }}$, L. Bellucci ${ }^{p}$, R. Berbeco ${ }^{c}$, J. Berdugo ${ }^{\mathrm{y}}$, P. Berges ${ }^{\mathrm{n}}$, B. Bertucci ${ }^{\text {af }}$, B.L. Betev ${ }^{\text {av }}$, S. Bhattacharya ${ }^{j}$, M. Biasini af ${ }^{\text {, A. Biland }}{ }^{\text {av }}$, J.J. Blaising ${ }^{\text {d, S.C. Blyth }}{ }^{\text {ag }}$, G.J. Bobbink ${ }^{\mathrm{b}}$, A. Böhm ${ }^{\mathrm{a}}$, L. Boldizsar ${ }^{\mathrm{m}}$, B. Borgia ${ }^{\text {ai }}$, D. Bourilkov ${ }^{\text {av }}$, M. Bourquin ${ }^{\text {s }}$, S. Braccini ${ }^{\text {s }}$, J.G. Branson ${ }^{\text {am }}$, V. Brigljevic ${ }^{\text {av }}$, F. Brochu ${ }^{\mathrm{d}}$, A. Buffini ${ }^{\mathrm{p}}$, A. Buijs ${ }^{\text {ar }}$, J.D. Burger ${ }^{\mathrm{n}}$, W.J. Burger ${ }^{\text {af }}$, X.D. Cai ${ }^{\mathrm{n}}$, M. Campanelli av , M. Capell ${ }^{n}$, G. Cara Romeo ${ }^{i}$, G. Carlino ${ }^{\mathrm{ab}}$, A.M. Cartacci ${ }^{\mathrm{p}}$, J. Casaus ${ }^{\mathrm{y}}$, G. Castellini ${ }^{\mathrm{p}}$, F. Cavallari ${ }^{\text {ai }}$, N. Cavallo ${ }^{\text {ak }}$, C. Cecchi ${ }^{\text {af }}$, M. Cerrada ${ }^{\mathrm{y}}$, F. Cesaroni ${ }^{\text {w }}$, M. Chamizo ${ }^{\text {s, }}$ Y.H. Chang ${ }^{\text {ax }}$, U.K. Chaturvedi ${ }^{\mathrm{r}}$, M. Chemarin ${ }^{\mathrm{x}}$, A. Chen ${ }^{\text {ax }}$, G. Chen ${ }^{\mathrm{g}}$, G.M. Chen ${ }^{\mathrm{g}}$, H.F. Chen ${ }^{\mathrm{t}}$, H.S. Chen ${ }^{\mathrm{g}}$, G. Chiefari ${ }^{\mathrm{ab}}$, L. Cifarelli ${ }^{\text {al }}$, F. Cindolo ${ }^{\text {i }}$, C. Civinini ${ }^{\text {p }}$, I. Clare ${ }^{\mathrm{n}}$, R. Clare ${ }^{\mathrm{n}}$, G. Coignet ${ }^{\mathrm{d}}$,

N. Colino ${ }^{\mathrm{y}}$, S. Costantini ${ }^{\mathrm{e}}, \mathrm{F}$. Cotorobai ${ }^{1}$, B. de la Cruz ${ }^{\mathrm{y}}, \mathrm{A}$. Csilling ${ }^{\mathrm{m}}$, S. Cucciarelli ${ }^{\text {af }}$, T.S. Dai ${ }^{n}$, J.A. van Dalen ${ }^{\text {ad }}$, R. D'Alessandro ${ }^{p}$, R. de Asmundis ${ }^{\text {ab }}$, P. Déglon ${ }^{\mathrm{s}}$, A. Degré ${ }^{\mathrm{d}}$, K. Deiters ${ }^{\text {at }}$, D. della Volpe ${ }^{\mathrm{ab}}$, E. Delmeire ${ }^{\mathrm{s}}$, P. Denes ${ }^{\text {ah }}$, F. DeNotaristefani ${ }^{\text {ai }}$, A. De Salvo ${ }^{\text {av }}$, M. Diemoz ${ }^{\text {ai }}$, M. Dierckxsens ${ }^{\text {b }}$, D. van Dierendonck ${ }^{\mathrm{b}}$, F. Di Lodovico ${ }^{\text {av }}$, C. Dionisi ${ }^{\text {ai }}$, M. Dittmar ${ }^{\text {av }}$, A. Dominguez $^{\text {am }}$, A. Doria ${ }^{\text {ab }}$, M.T. Dova ${ }^{\mathrm{r}, 5}$, D. Duchesneau $^{\mathrm{d}}$, D. Dufournaud ${ }^{\mathrm{d}}$, P. Duinker ${ }^{\mathrm{b}}$, I. Duran ${ }^{\text {an }}$, H. El Mamouni ${ }^{\mathrm{x}}$, A. Engler ${ }^{\text {ag }}$, F.J. Eppling ${ }^{\text {n }}$, F.C. Erné ${ }^{\mathrm{b}}$, P. Extermann ${ }^{\text {s }}$, M. Fabre ${ }^{\text {at }}$, R. Faccini ${ }^{\text {ai }}$, M.A. Falagan ${ }^{y}$, S. Falciano ${ }^{\text {ai, }}$, A. Favara ${ }^{\mathrm{q}}$, J. Fay ${ }^{\mathrm{x}}$, O. Fedin ${ }^{\text {aj }}$, M. Felcini ${ }^{\text {av }}$, T. Ferguson ${ }^{\text {ag }}$, F. Ferroni ${ }^{\text {ai }}$, H. Fesefeldt ${ }^{\text {a }}$, E. Fiandrini ${ }^{\text {af }}$, J.H. Field ${ }^{\text {s }}$, F. Filthaut ${ }^{\text {q }}$, P.H. Fisher ${ }^{\text {n }}$, I. Fisk ${ }^{\text {am }}$, G. Forconi ${ }^{n}$, K. Freudenreich ${ }^{\text {av }}$, C. Furetta ${ }^{z}$, Yu. Galaktionov ${ }^{\text {aa, }}$, 
S.N. Ganguli ${ }^{\text {j }, ~ P . ~ G a r c i a-A b i a ~}{ }^{\mathrm{e}}$, M. Gataullin ${ }^{\text {ae }}$, S.S. Gau ${ }^{\mathrm{k}}$, S. Gentile ${ }^{\text {ai, }}$,

N. Gheordanescu ${ }^{1}$, S. Giagu ${ }^{\text {ai }}$, Z.F. Gong ${ }^{\mathrm{t}}$, G. Grenier ${ }^{\mathrm{x}}$, O. Grimm ${ }^{\mathrm{av}}$,

M.W. Gruenewald ${ }^{\text {h }}$, M. Guida ${ }^{\text {al }}$, R. van Gulik ${ }^{\text {b }}$, V.K. Gupta ${ }^{\text {ah }}$, A. Gurtu ${ }^{\text {j }}$,

L.J. Gutay ${ }^{\text {as }}$, D. Haas ${ }^{\text {e, }}$ A. Hasan ${ }^{\text {ac }}$, D. Hatzifotiadou ${ }^{i}$, T. Hebbeker ${ }^{\text {h }}$, A. Hervé ${ }^{\text {, }}$,

P. Hidas ${ }^{\mathrm{m}}$, J. Hirschfelder ${ }^{\text {ag }}$, H. Hofer ${ }^{\text {av }}$, G. Holzner ${ }^{\text {av }}$, H. Hoorani ${ }^{\text {ag }}$, S.R. Hou ${ }^{\text {ax }}$,

Y. Hu ${ }^{\text {ad }}$, I. Iashvili ${ }^{\text {au }}$, B.N. Jin ${ }^{\mathrm{g}}$, L.W. Jones ${ }^{\mathrm{c}}$, P. de Jong ${ }^{\mathrm{b}}$, I. Josa-Mutuberría ${ }^{\mathrm{y}}$, R.A. Khan ${ }^{\text {r }}$, M. Kaur ${ }^{\text {r,6 }}$, M.N. Kienzle-Focacci ${ }^{\text {s }}$, D. Kim ${ }^{\text {ai }}$, J.K. Kim ${ }^{\text {ap }}$, J. Kirkby ${ }^{\text {q }}$, D. Kiss ${ }^{\mathrm{m}}$, W. Kittel ${ }^{\text {ad }}$, A. Klimentov ${ }^{\text {n,aa }}$, A.C. König ${ }^{\text {ad }}$, A. Kopp ${ }^{\text {au }}$, V. Koutsenko ${ }^{\text {naa }}$,

M. Kräber ${ }^{\text {av }}$, R.W. Kraemer ${ }^{\text {ag }}$, W. Krenz ${ }^{\text {a }}$, A. Krüger ${ }^{\text {au }}$, A. Kunin ${ }^{\text {n,aa }}$, P. Ladron de Guevara ${ }^{y}$, I. Laktineh ${ }^{\mathrm{x}}$, G. Landi ${ }^{\mathrm{p}}$, K. Lassila-Perini ${ }^{\text {av }}$, M. Lebeau ${ }^{\mathrm{q}}$, A. Lebedev ${ }^{n}$, P. Lebrun ${ }^{x}$, P. Lecomte ${ }^{\text {av }}$, P. Lecoq ${ }^{\text {q }}$, P. Le Coultre ${ }^{\text {av }}$, H.J. Lee ${ }^{\text {h }}$, J.M. Le Goff ${ }^{\mathrm{q}}$, R. Leiste ${ }^{\text {au }}$, E. Leonardi ${ }^{\text {ai }}$, P. Levtchenko ${ }^{\text {aj }}, \mathrm{C} \mathrm{Li}^{\mathrm{t}}$, S. Likhoded ${ }^{\text {au }}$, C.H. Lin ${ }^{a x}$, W.T. Lin $^{a x}$, F.L. Linde ${ }^{b}$, L. Lista ${ }^{a b}$, Z.A. Liu ${ }^{g}$, W. Lohmann ${ }^{\text {au }}$, E. Longo ${ }^{\text {ai }}$, Y.S. Lu ${ }^{\text {g }}$, K. Lübelsmeyer ${ }^{\text {a }}$, C. Luci ${ }^{\text {q,ai }}$, D. Luckey ${ }^{\text {n }}$, L. Lugnier ${ }^{x}$, L. Luminari ai, W. Lustermann ${ }^{\text {av }}$, W.G. Ma ${ }^{\mathrm{t}}$, M. Maity ${ }^{\mathrm{j}}$, L. Malgeri ${ }^{\mathrm{q}}$, A. Malinin ${ }^{\mathrm{q}}$, C. Maña ${ }^{y}$, D. Mangeol ${ }^{\text {ad }}$, J. Mans ${ }^{\text {ah }}$, P. Marchesini ${ }^{\text {av }}$, G. Marian ${ }^{\circ}$, J.P. Martin ${ }^{\mathrm{x}}$, F. Marzano ${ }^{\text {ai }}$, K. Mazumdar ${ }^{\mathrm{j}}$, R.R. McNeil ${ }^{\mathrm{f}}$, S. Mele ${ }^{\mathrm{q}}$, L. Merola ${ }^{\mathrm{ab}}$, M. Meschini ${ }^{\text {p }}$, W.J. Metzger ${ }^{\text {ad }}$, M. von der Mey ${ }^{\text {a }}$, A. Mihul ${ }^{1}$, H. Milcent ${ }^{q}$, G. Mirabelli ai , J. Mnich ${ }^{\mathrm{q}}$, G.B. Mohanty ${ }^{\mathrm{j}}$, P. Molnar ${ }^{\mathrm{h}}$, T. Moulik ${ }^{\mathrm{j}}$, G.S. Muanza ${ }^{\text {x }}$, A.J.M. Muijs ${ }^{b}$, B. Musicar ${ }^{\text {am }}$, M. Musy ${ }^{\text {ai }}$, M. Napolitano ${ }^{\text {ab }}$, F. Nessi-Tedaldi ${ }^{\text {av }}$, H. Newman ${ }^{\text {ae }}$, T. Niessen ${ }^{\text {a }}$, A. Nisati ${ }^{\text {ai }}$, H. Nowak ${ }^{\text {au }}$, G. Organtini ${ }^{\text {ai }}$, A. Oulianov ${ }^{\text {aa }}$, C. Palomares ${ }^{\text {y }}$, D. Pandoulas ${ }^{\text {a }}$, S. Paoletti ${ }^{\text {ai, }}$, P. Paolucci ${ }^{\text {ab }}$, R. Paramatti ${ }^{\text {ai }}$, H.K. Park ${ }^{\text {ag }}$, I.H. Park ${ }^{\text {ap }}$, G. Passaleva ${ }^{\text {q }}$, S. Patricelli ${ }^{\text {ab }}$, T. Paul $^{\text {k }}$, M. Pauluzzi ${ }^{\text {af }}$, C. Paus ${ }^{\mathrm{q}}$, F. Pauss ${ }^{\text {av }}$, M. Pedace ${ }^{\text {ai }}$, S. Pensotti $^{z}$, D. Perret-Gallix ${ }^{d}$, B. Petersen ${ }^{\text {ad }}$, D. Piccolo ${ }^{\text {ab }}$, F. Pierella ${ }^{i}$, M. Pieri $^{p}$, P.A. Piroué ${ }^{\text {ah }}$, E. Pistolesi ${ }^{\mathrm{z}}$, V. Plyaskin ${ }^{\text {aa }}$, M. Pohl ${ }^{\mathrm{s}}$, V. Pojidaev ${ }^{\text {aa, }}{ }^{\text {, H. Postema }}{ }^{\mathrm{n}}$, J. Pothier ${ }^{\text {q }}$, D.O. Prokofiev ${ }^{\text {as }}$, D. Prokofiev ${ }^{\text {aj }}$, J. Quartieri ${ }^{\text {al }}$, G. Rahal-Callot ${ }^{\text {av, }}{ }^{\text {, }}$, M.A. Rahaman ${ }^{j}$, P. Raics ${ }^{\circ}$, N. Raja ${ }^{j}$, R. Ramelli ${ }^{\text {av }}$, P.G. Rancoita ${ }^{z}$, A. Raspereza ${ }^{\text {au }}$, G. Raven ${ }^{\text {am }}$, P. Razis ${ }^{\text {ac }}$, D. Ren ${ }^{\text {av }}$, M. Rescigno ${ }^{\text {ai }}$, S. Reucroft ${ }^{k}$, S. Riemann ${ }^{\text {au }}$, K. Riles ${ }^{c}$, A. Robohm ${ }^{\text {av }}$, J. Rodin ${ }^{\text {aq }}$, B.P. Roe ${ }^{c}$, L. Romero ${ }^{y}$, A. Rosca ${ }^{\text {h }}$, S. Rosier-Lees ${ }^{d}$, J.A. Rubio ${ }^{\mathrm{q}}$, G. Ruggiero ${ }^{\mathrm{p}}$, D. Ruschmeier ${ }^{\mathrm{h}}$, H. Rykaczewski ${ }^{\text {av }}$, S. Saremi ${ }^{\text {f }}$, S. Sarkar ${ }^{\text {ai }}$, J. Salicio ${ }^{\text {q }}$, E. Sanchez ${ }^{\text {q }}$, M.P. Sanders ${ }^{\text {ad }}$, M.E. Sarakinos ${ }^{\text {u }}$, C. Schäfer ${ }^{\text {q }, ~ V . ~ S c h e g e l s k y ~}{ }^{\text {aj }, ~ S . ~ S c h m i d t-K a e r s t ~}{ }^{\text {a }}$, D. Schmitz ${ }^{\text {a }}$, H. Schopper ${ }^{\text {aw }}$, D.J. Schotanus ${ }^{\text {ad }}$, G. Schwering ${ }^{\text {a }}$, C. Sciacca ${ }^{\text {ab }}$, D. Sciarrino ${ }^{\text {s }}$, A. Seganti ${ }^{\text {i }}$, L. Servoli ${ }^{\text {af }}$, S. Shevchenko ${ }^{\text {ae }}$, N. Shivarov ${ }^{\text {ao }}$, V. Shoutko ${ }^{\text {aa }}$, E. Shumilov ${ }^{\text {aa }}$, A. Shvorob ${ }^{\text {ae }}$, T. Siedenburg ${ }^{\text {a }}$, D. Son ${ }^{\text {ap }}$, B. Smith ${ }^{\text {ag }}$, P. Spillantini ${ }^{p}$, M. Steuer ${ }^{n}$, D.P. Stickland ${ }^{\text {ah }}$, A. Stone ${ }^{\text {f }}$, B. Stoyanov ${ }^{\text {ao }}$, A. Straessner ${ }^{\text {a }}$, K. Sudhakar ${ }^{\text {j, }}$,

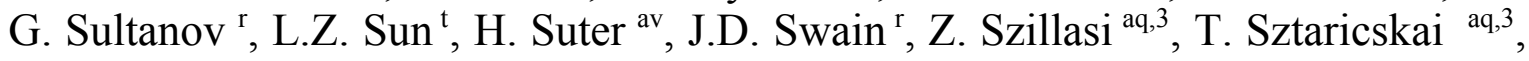


X.W. Tang ${ }^{g}$, L. Tauscher ${ }^{\mathrm{e}}$, L. Taylor ${ }^{\mathrm{k}}$, B. Tellili ${ }^{\mathrm{x}}$, C. Timmermans ${ }^{\mathrm{ad}}$, Samuel C.C. Ting ${ }^{n}$, S.M. Ting ${ }^{n}$, S.C. Tonwar ${ }^{j}$, J. Tóth ${ }^{\mathrm{m}}$, C. Tully ${ }^{\mathrm{q}}$, K.L. Tung ${ }^{\mathrm{g}}$, Y. Uchida ${ }^{\mathrm{n}}$, J. Ulbricht ${ }^{\text {av }}$, E. Valente ${ }^{\text {ai }}$, G. Vesztergombi ${ }^{\mathrm{m}}$, I. Vetlitsky ${ }^{\text {aa }}$, D. Vicinanza $^{\text {al }}$, G. Viertel ${ }^{\text {av }}$, S. Villa ${ }^{\mathrm{k}}$, M. Vivargent ${ }^{\mathrm{d}}, \mathrm{S}$. Vlachos ${ }^{\mathrm{e}}$, I. Vodopianov ${ }^{\text {aj }}$, H. Vogel ${ }^{\text {ag }}$, H. Vogt ${ }^{\text {au}}$, I. Vorobiev ${ }^{\text {aa }}$, A.A. Vorobyov ${ }^{\text {aj, }}$, A. Vorvolakos ${ }^{\text {ac }}$, M. Wadhwa ${ }^{\mathrm{e}}$, W. Wallraff ${ }^{\mathrm{a}}$, M. Wang ${ }^{\mathrm{n}}$, X.L. Wang ${ }^{\mathrm{t}}$, Z.M. Wang ${ }^{\mathrm{t}}$, A. Weber ${ }^{\text {a }}$, M. Weber ${ }^{\text {a }}$, P. Wienemann ${ }^{\text {a }}$, H. Wilkens ${ }^{\text {ad }}$, S.X. Wu ${ }^{\text {n }}$, S. Wynhoff ${ }^{\mathrm{q}}$, L. Xia ${ }^{\text {ae }}$, Z.Z. Xu ${ }^{\mathrm{t}}$, J. Yamamoto ${ }^{\mathrm{c}}$, B.Z. Yang ${ }^{\mathrm{t}}$, C.G. Yang ${ }^{\mathrm{g}}$, H.J. Yang ${ }^{\mathrm{g}}$, M. Yang ${ }^{g}$, J.B. Ye ${ }^{t}$, S.C. Yeh ${ }^{\text {ay }}$, An. Zalite ${ }^{\text {aj }}$, Yu. Zalite ${ }^{\text {aj }}$, Z.P. Zhang ${ }^{t}$, G.Y. Zhu ${ }^{\text {g }}$,

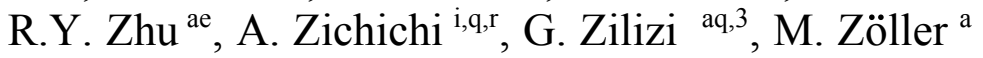

${ }^{a}$ I. Physikalisches Institut, RWTH, D-52056 Aachen, Germany,

and III. Physikalisches Institut, RWTH, D-52056 Aachen, Germany ${ }^{I}$

${ }^{\mathrm{b}}$ National Institute for High Energy Physics, NIKHEF, and University of Amsterdam, NL-1009 DB Amsterdam, The Netherlands

${ }^{\mathrm{c}}$ University of Michigan, Ann Arbor, MI 48109, USA

d Laboratoire d'Annecy-le-Vieux de Physique des Particules, LAPP, IN2P3-CNRS, BP 110, F-74941 Annecy-le-Vieux CEDEX, France

${ }^{\mathrm{e}}$ Institute of Physics, University of Basel, CH-4056 Basel, Switzerland

${ }^{\mathrm{f}}$ Louisiana State University, Baton Rouge, LA 70803, USA

${ }^{\mathrm{g}}$ Institute of High Energy Physics, IHEP, 100039 Beijing, China ${ }^{7}$

${ }^{\mathrm{h}}$ Humboldt University, D-10099 Berlin, Germany ${ }^{1}$

${ }^{\mathrm{i}}$ University of Bologna and INFN-Sezione di Bologna, I-40126 Bologna, Italy

${ }^{\mathrm{j}}$ Tata Institute of Fundamental Research, Bombay 400 005, India

${ }^{\mathrm{k}}$ Northeastern University, Boston, MA 02115, USA

${ }^{1}$ Institute of Atomic Physics and University of Bucharest, R-76900 Bucharest, Romania

${ }^{\mathrm{m}}$ Central Research Institute for Physics of the Hungarian Academy of Sciences, H-1525 Budapest 114, Hungary ${ }^{2}$

${ }^{\mathrm{n}}$ Massachusetts Institute of Technology, Cambridge, MA 02139, USA

${ }^{\circ}$ KLTE-ATOMKI, H-4010 Debrecen, Hungary ${ }^{3}$

${ }^{\mathrm{p}}$ INFN Sezione di Firenze and University of Florence, I-50125 Florence, Italy

${ }^{\mathrm{q}}$ European Laboratory for Particle Physics, CERN, CH-1211 Geneva 23, Switzerland

${ }^{\mathrm{r}}$ World Laboratory, FBLJA Project, CH-1211 Geneva 23, Switzerland

${ }^{\mathrm{s}}$ University of Geneva, CH-1211 Geneva 4, Switzerland

${ }^{t}$ Chinese University of Science and Technology, USTC, Hefei, Anhui 230 029, China ${ }^{7}$

" SEFT, Research Institute for High Energy Physics, P.O. Box 9, SF-00014 Helsinki, Finland

${ }^{v}$ University of Lausanne, CH-1015 Lausanne, Switzerland

${ }^{\mathrm{w}}$ INFN-Sezione di Lecce and Universitá Degli Studi di Lecce, I-73100 Lecce, Italy

${ }^{\mathrm{x}}$ Institut de Physique Nucléaire de Lyon, IN2P3-CNRS, Université Claude Bernard, F-69622 Villeurbanne, France

${ }^{y}$ Centro de Investigaciones Energéticas, Medioambientales y Tecnologícas, CIEMAT, E-28040 Madrid, Spain ${ }^{4}$

${ }^{\mathrm{z}}$ INFN-Sezione di Milano, I-20133 Milan, Italy

aa Institute of Theoretical and Experimental Physics, ITEP, Moscow, Russia

${ }^{\mathrm{ab}}$ INFN-Sezione di Napoli and University of Naples, I-80125 Naples, Italy

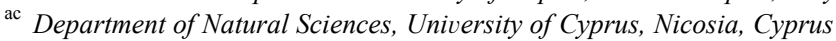

${ }^{\text {ad }}$ University of Nijmegen and NIKHEF, NL-6525 ED Nijmegen, The Netherlands

${ }^{\text {ae }}$ California Institute of Technology, Pasadena, CA 91125, USA

af INFN-Sezione di Perugia and Universitá Degli Studi di Perugia, I-06100 Perugia, Italy

ag Carnegie Mellon University, Pittsburgh, PA 15213, USA

ah Princeton University, Princeton, NJ 08544, USA

ai INFN-Sezione di Roma and University of Rome, 'La Sapienza', I-00185 Rome, Italy

${ }^{\text {aj }}$ Nuclear Physics Institute, St. Petersburg, Russia

${ }^{\mathrm{ak}}$ INFN-Sezione di Napoli and University of Potenza, I-85100 Potenza, Italy

${ }^{\text {al }}$ University and INFN, Salerno, I-84100 Salerno, Italy

am University of California, San Diego, CA 92093, USA

${ }^{a n}$ Dept. de Fisica de Particulas Elementales, Univ. de Santiago, E-15706 Santiago de Compostela, Spain

ao Bulgarian Academy of Sciences, Central Lab. of Mechatronics and Instrumentation, BU-1113 Sofia, Bulgaria 
ap Laboratory of High Energy Physics, Kyungpook National University, 702-701 Taegu, South Korea

aq University of Alabama, Tuscaloosa, AL 35486, USA

${ }^{\text {ar }}$ Utrecht University and NIKHEF, NL-3584 CB Utrecht, The Netherlands

as Purdue University, West Lafayette, IN 47907, USA

${ }^{\text {at }}$ Paul Scherrer Institut, PSI, CH-5232 Villigen, Switzerland

au DESY, D-15738 Zeuthen, Germany

av Eidgenössische Technische Hochschule, ETH Zürich, CH-8093 Zürich, Switzerland

${ }^{\text {aw }}$ University of Hamburg, D-22761 Hamburg, Germany

${ }^{a x}$ National Central University, Chung-Li, Taiwan, ROC

ay Department of Physics, National Tsing Hua University, Taiwan, ROC

Received 8 May 2000; accepted 20 July 2000

Editor: K. Winter

\begin{abstract}
The measurements of hadron and lepton-pair production cross sections and leptonic forward-backward asymmetries performed with the L3 detector at centre-of-mass energies between $130 \mathrm{GeV}$ and $189 \mathrm{GeV}$ are used to search for new physics phenomena such as: contact interactions, exchange of virtual leptoquarks, scalar quarks and scalar neutrinos, effects of $\mathrm{TeV}$ strings in models of quantum gravity with large extra dimensions and non-zero sizes of the fermions. No evidence for these phenomena is found and new limits on their parameters are set. (C) 2000 Elsevier Science B.V. All rights reserved.
\end{abstract}

\section{Introduction}

The study of fermion-pair production, $\mathrm{e}^{+} \mathrm{e}^{-} \rightarrow \overline{f f}$, at centre-of-mass energies well above the $\mathrm{Z}$ resonance allows to look for physics beyond the Standard Model. The successful running of LEP in 1997 and 1998 at energies of $182.7 \mathrm{GeV}$ and $188.7 \mathrm{GeV}$, and the tenfold increase of luminosity compared to our previous searches [1,2], improves substantially the sensitivity to new physics phenomena.

The results presented in this paper are based on analyses of our measurements of hadronic and leptonic cross sections and leptonic forward-backward

\footnotetext{
E-mail address: salvatore.mele@cern.ch (S. Mele).

${ }^{1}$ Supported by the German Bundesministerium für Bildung, Wissenschaft, Forschung und Technologie.

${ }^{2}$ Supported by the Hungarian OTKA fund under contract numbers T019181, F023259 and T024011.

${ }^{3}$ Also supported by the Hungarian OTKA fund under contract numbers T22238 and T026178.

${ }^{4}$ Supported also by the Comisión Interministerial de Ciencia y Tecnología.

${ }^{5}$ Also supported by CONICET and Universidad Nacional de La Plata, CC 67, 1900 La Plata, Argentina.

${ }^{6}$ Also supported by Panjab University, Chandigarh-160014, India.

${ }^{7}$ Supported by the National Natural Science Foundation of China.
}

asymmetries [3-5]. The measurements in all channels are used to search for four-fermion contact interactions. The virtual exchange of leptoquarks and scalar quarks is investigated using our hadron cross section measurements. The effects of scalar neutrino exchange are looked for in all leptonic channels. Limits on contact interactions, and on leptoquark, scalar quark and scalar neutrino couplings have been presented also by other LEP collaborations [6-8]. The effects of $\mathrm{TeV}$ strings, predicted recently $[9,10]$ in theories of quantum gravity with extra dimensions [11-13], are searched for in Bhabha scattering. This is an extension of the searches for low scale gravity in fermion-pair production at LEP [8,14-17]. Furthermore, a form factor ansatz is used to estimate the size of leptons and quarks.

\section{Data and analysis method}

Measurements of cross sections and forwardbackward asymmetries for the reactions $\mathrm{e}^{+} \mathrm{e}^{-} \rightarrow \overline{f f}$ have been performed with the L3 detector [18-22] at centre-of-mass energies, $\sqrt{s}$, of $130.0 \mathrm{GeV}, 136.1$ $\mathrm{GeV}, 161.3 \mathrm{GeV}, 172.3 \mathrm{GeV}, 182.7 \mathrm{GeV}$ and $188.7 \mathrm{GeV}$ [3-5]. They correspond to an integrated luminosity of $265.4 \mathrm{pb}^{-1}$. 
For the $\mathrm{e}^{+} \mathrm{e}^{-}$final state both leptons have to be in the polar angular range $44^{\circ}<\theta<136^{\circ}$, where $\theta$ is the angle between the incoming electron and the outgoing lepton. Muon- and tau-pair candidates are selected with both leptons in the fiducial volume given by $|\cos \theta|<0.9$ and $|\cos \theta|<0.92$, respectively. Hadron events are selected in the full solid angle.

In total 28470 hadron events and 9417 lepton-pair events are selected. A minimum effective centre-ofmass energy, $\sqrt{s_{\min }^{\prime}}$, or a maximum acollinearity angle in the Bhabha channel, are required to select events without substantial energy loss due to initial state radiation. The remaining samples, which are studied in this paper, contain in total 7785 hadron and 7704 lepton-pair events.

The measurements of total cross sections and leptonic forward-backward asymmetries are analysed in terms of new physics, which will manifest itself as deviations from the Standard Model predictions. The contributions of contact interactions, leptoquarks and scalar quarks are included directly into the improved Born cross section calculated with the program ZFITTER [23] and are convoluted to account for QED radiative corrections. For the analyses including the $\mathrm{e}^{+} \mathrm{e}^{-}$final state, i.e. contact interactions, scalar neutrinos, TeV strings and form factors, the effects of new phenomena are computed with dedicated programs in the improved Born approximation, taking into account QED radiative corrections. For contact interactions where both approaches are used, the results agree well with each other.

The measurements are compared to the predictions of the Standard Model [24] as calculated using the ZFITTER and TOPAZ0 [25] programs with the following parameters [26-30]: $m_{\mathrm{Z}}=91.190 \mathrm{GeV}$, $\alpha_{s}\left(m_{\mathrm{Z}}^{2}\right)=0.119, m_{\mathrm{t}}=173.8 \mathrm{GeV}, \Delta \alpha_{\text {had }}^{(5)}=0.02804$, and $m_{\mathrm{H}}=150 \mathrm{GeV}$. The results of our analyses are not sensitive to small variations of these parameters. The theoretical uncertainties on the Standard Model predictions are estimated to be below $1 \%$ except for large angle Bhabha scattering where the uncertainty is $2 \%$ [25].

The measurements show no statistically significant deviations from the Standard Model expectations. In their absence, limits at 95\% confidence level on the contributions of new physics are determined by integrating the log-likelihood functions in the physically allowed range of the parameters describing new physics phenomena, assuming a uniform prior distribution. The statistical errors and systematic uncertainties of the measurements [4,5], as well as the theory uncertainties given above, are combined in quadrature for all analyses.

\section{Four-fermion contact interactions}

Four-fermion contact interactions offer a general framework for describing interactions beyond the Standard Model. They are characterised by a coupling strength, $g$, and by an energy scale, $\Lambda$, which can be viewed as the typical mass of new heavy particles being exchanged. At energies much lower than $\Lambda$, the exchange of virtual new particles is described by an effective Lagrangian [31]:

$\mathscr{L}=\frac{1}{1+\delta_{\mathrm{e} f}} \sum_{i, j=\mathrm{L}, \mathrm{R}} \eta_{i j} \frac{g^{2}}{\Lambda_{i j}^{2}}\left(\overline{\mathrm{e}}_{i} \gamma^{\mu} \mathrm{e}_{i}\right)\left(\bar{f}_{j} \gamma_{\mu} f_{j}\right)$,

where $\mathrm{e}_{i}$ and $f_{j}$ denote the left- and right-handed initial-state electron and final-state fermion fields. The Kronecker symbol, $\delta_{\mathrm{e} f}$, is zero except for the $\mathrm{e}^{+} \mathrm{e}^{-}$final state where it is one. The parameters $\eta_{i j}$ define the contact interaction model by choosing the helicity amplitudes which contribute to the reaction

Table 1

Models of contact interaction considered. The parameters $\eta_{i j}(i, j=\mathrm{L}, \mathrm{R})$ define to which helicity amplitudes the contact interactions contribute

\begin{tabular}{lrrrrrrrrr}
\hline Model & LL & RR & LR & RL & VV & AA & V0 & A0 & LL-RR \\
\hline$\eta_{\mathrm{LL}}$ & \pm 1 & 0 & 0 & 0 & \pm 1 & \pm 1 & \pm 1 & 0 & \pm 1 \\
$\eta_{\mathrm{RR}}$ & 0 & \pm 1 & 0 & 0 & \pm 1 & \pm 1 & \pm 1 & 0 & $\mp 1$ \\
$\eta_{\mathrm{LR}}$ & 0 & 0 & \pm 1 & 0 & \pm 1 & $\mp 1$ & 0 & \pm 1 & 0 \\
$\eta_{\mathrm{RL}}$ & 0 & 0 & 0 & \pm 1 & \pm 1 & $\mp 1$ & 0 & \pm 1 & 0 \\
\hline
\end{tabular}


Table 2

The one-sided $95 \%$ confidence level lower limits on the parameter $\Lambda$ of contact interaction derived from fits to lepton-pair cross sections and asymmetries. The limits $\Lambda_{+}$and $\Lambda_{-}$given in TeV correspond to the upper and lower signs of the parameters $\eta_{i j}$ in Table 1

\begin{tabular}{|c|c|c|c|c|c|c|c|c|}
\hline \multirow[t]{2}{*}{ Model } & \multicolumn{2}{|l|}{$\mathrm{e}^{+} \mathrm{e}^{-}$} & \multicolumn{2}{|c|}{$\mu^{+} \mu^{-}$} & \multicolumn{2}{|c|}{$\tau^{+} \tau^{-}$} & \multicolumn{2}{|c|}{$\ell^{+} \ell^{-}$} \\
\hline & $\Lambda_{-}$ & $\Lambda_{+}$ & $\Lambda_{-}$ & $\Lambda_{+}$ & $\Lambda_{-}$ & $\Lambda_{+}$ & $\Lambda_{-}$ & $\Lambda_{+}$ \\
\hline LL & 4.9 & 4.3 & 3.8 & 8.5 & 4.7 & 5.4 & 5.2 & 9.0 \\
\hline LR & 5.8 & 5.1 & 2.0 & 6.5 & 1.8 & 3.7 & 6.4 & 6.3 \\
\hline RL & 5.8 & 5.1 & 2.0 & 6.5 & 1.8 & 3.7 & 6.4 & 6.3 \\
\hline $\mathrm{AA}$ & 5.4 & 6.8 & 6.7 & 9.7 & 5.5 & 8.6 & 7.1 & 12.4 \\
\hline V0 & 6.8 & 6.3 & 5.4 & 11.7 & 6.6 & 7.3 & 7.3 & 12.5 \\
\hline A0 & 8.0 & 7.5 & 2.1 & 9.0 & 1.9 & 5.0 & 9.0 & 8.9 \\
\hline LL-RR & 3.0 & 3.0 & 3.5 & 4.4 & 2.9 & 3.3 & 3.8 & 4.6 \\
\hline
\end{tabular}

$\mathrm{e}^{+} \mathrm{e}^{-} \rightarrow \overline{f f}$. The value of $g / \Lambda$ determines the size of the expected effects. By convention $g^{2} / 4 \pi$ is cho- sen to be 1 and $\left|\eta_{i j}\right|=1$ or $\left|\eta_{i j}\right|=0$, leaving the energy scale $\Lambda$ as a free parameter. The helicity

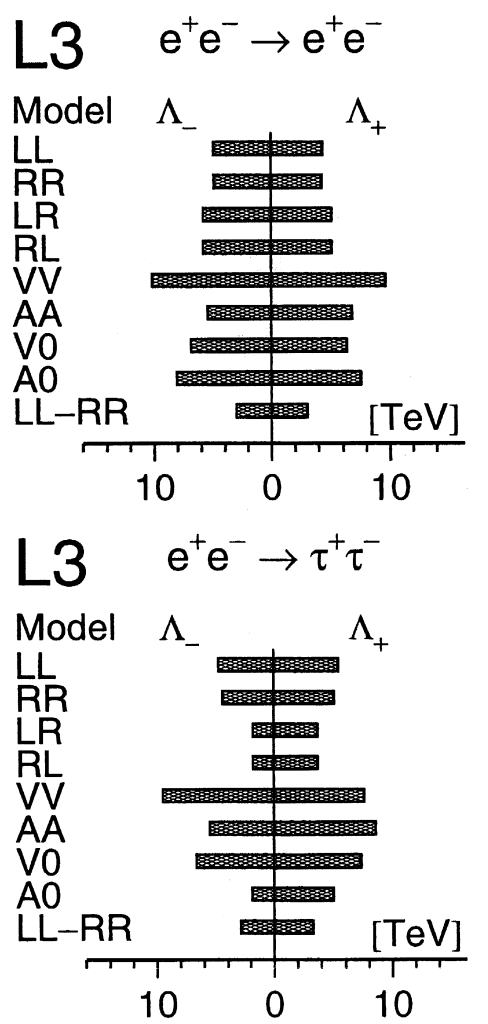

L3 $e^{+} e^{-} \rightarrow \mu^{+} \mu^{-}$

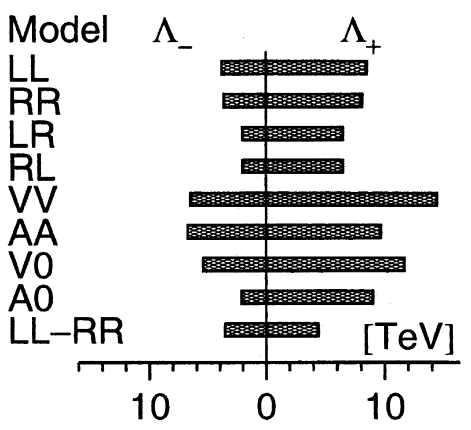

L3 $\mathrm{e}^{+} \mathrm{e}^{-} \rightarrow \mathrm{I}^{+} \mathrm{I}^{-}$

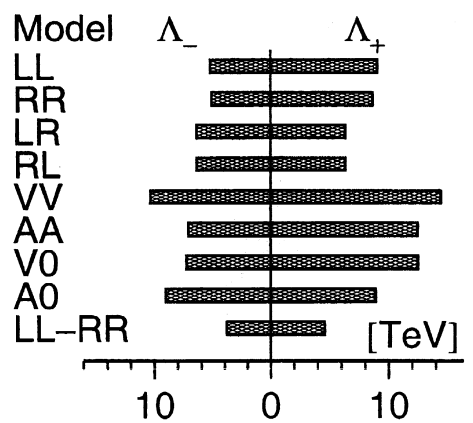

Fig. 1. One-sided 95\% confidence level lower limits on the scales $\Lambda_{+}$and $\Lambda_{-}$for contact interactions in leptonic channels. The limits correspond to the values given in Table 2 . 
Table 3

The one-sided $95 \%$ confidence level lower limits on the parameter $\Lambda$ of contact interaction derived from fits to hadron cross sections, and for all fermions combined. The limits $\Lambda_{+}$and $\Lambda_{-}$given in TeV correspond to the upper and lower signs of the parameters $\eta_{i j}$ in Table 1

\begin{tabular}{|c|c|c|c|c|c|c|c|c|}
\hline \multirow[t]{2}{*}{ Model } & \multicolumn{2}{|l|}{$\mathrm{q} \overline{\mathrm{q}}$} & \multicolumn{2}{|l|}{$\mathrm{uu}$} & \multicolumn{2}{|l|}{$\mathrm{d} \overline{\mathrm{d}}$} & \multicolumn{2}{|l|}{$\overline{f f}$} \\
\hline & $\Lambda_{-}$ & $\Lambda_{+}$ & $\Lambda_{-}$ & $\Lambda_{+}$ & $\Lambda_{-}$ & $\Lambda_{+}$ & $\Lambda_{-}$ & $\Lambda_{+}$ \\
\hline LL & 2.8 & 4.2 & 4.1 & 7.0 & 6.5 & 3.9 & 5.5 & 8.3 \\
\hline $\mathrm{RR}$ & 3.8 & 3.1 & 3.6 & 1.5 & 1.8 & 2.9 & 4.9 & 9.0 \\
\hline LR & 3.5 & 3.3 & 2.7 & 1.9 & 2.1 & 2.5 & 5.9 & 6.1 \\
\hline RL & 4.6 & 2.5 & 2.4 & 2.2 & 2.8 & 1.8 & 6.0 & 7.9 \\
\hline VV & 5.5 & 4.2 & 5.8 & 9.8 & 2.2 & 4.6 & 9.8 & 15.0 \\
\hline $\mathrm{AA}$ & 3.8 & 6.1 & 5.0 & 7.4 & 7.5 & 5.1 & 7.6 & 11.3 \\
\hline V0 & 3.7 & 4.4 & 5.2 & 9.2 & 7.7 & 4.7 & 7.2 & 12.1 \\
\hline A0 & 5.2 & 3.1 & 3.2 & 2.3 & 2.9 & 2.5 & 8.3 & 10.0 \\
\hline LL-RR & 3.2 & 5.1 & 3.2 & 2.3 & 2.1 & 3.6 & 3.5 & 5.5 \\
\hline
\end{tabular}

combinations of the specific models considered are defined in Table 1. Atomic physics parity violation experiments probe with high precision the couplings of electrons to quarks of the first family, and place

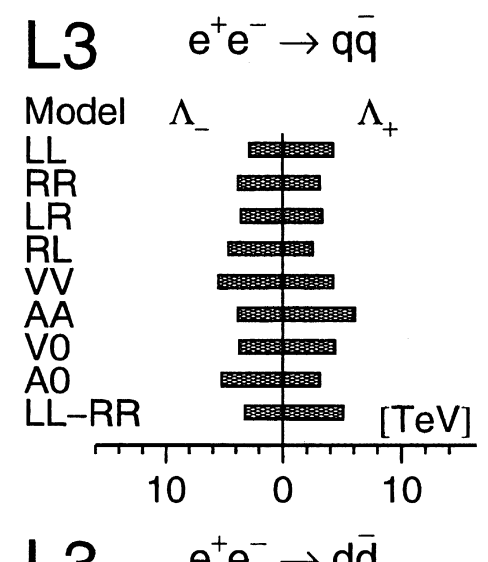

L3 $e^{+} e^{-} \rightarrow u \bar{u}$

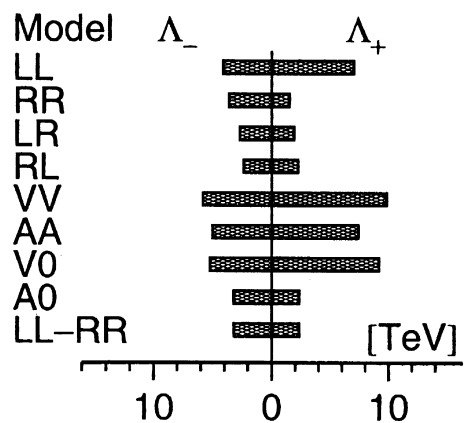

L3

$\mathrm{e}^{+} \mathrm{e}^{-} \rightarrow \mathrm{d} \overline{\mathrm{d}}$

L3 $\mathrm{e}^{+} \mathrm{e}^{-} \rightarrow \mathrm{ff}$
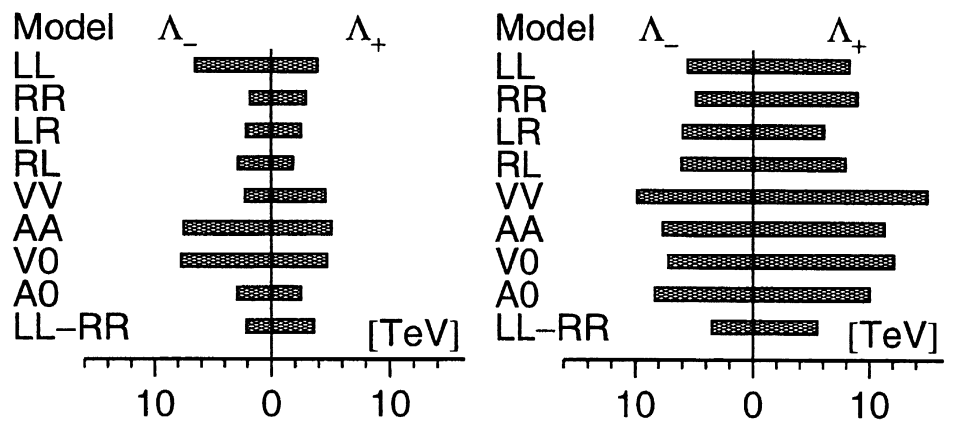

Fig. 2. One-sided $95 \%$ confidence level lower limits on the scales $\Lambda_{+}$and $\Lambda_{-}$for contact interactions in hadronic channels and in all channels combined. The limits correspond to the values given in Table 3 . 
severe constraints on the scale $\Lambda$ of the order of 15 $\mathrm{TeV}$ [32]. The VV, AA, V0 and A0 models are parity conserving and hence are not constrained by such measurements.

The four-fermion contact interactions for the different types of final-state fermions are tested separately as well as for all flavours combined and lower limits on the scale $\Lambda$ are derived. The lower limits on $\Lambda$ obtained from lepton-pair final states are summarised in Table 2 and Fig. 1. It is important to note that the pure leptonic case is only accessible at LEP.

For hadronic final states the cases where the contact interactions affect either all flavours at the same time, or only one flavour of up-type or downtype quarks, are analyzed. The results are given in Table 3 and depicted in Fig. 2, together with the combined results for all charged fermions. Similar limits are obtained from studies of deep inelastic scattering at HERA $[33,34]$ and proton-antiproton collisions at the TEVATRON [35,36].

\section{Leptoquarks}

Leptoquarks couple to quark-lepton pairs from the same family, preserving the baryon number $B$ and the lepton number $L$. Leptoquarks carry fermion numbers, $F=L+3 B$. Following the notation in Ref. [37], scalar leptoquarks $S_{I}$ and vector leptoquarks $V_{I}$ are indicated based on spin and isospin $I$. Isomultiplets with different hypercharges are denoted by an additional tilde.

In the process $\mathrm{e}^{+} \mathrm{e}^{-} \rightarrow$ hadrons, leptoquarks of the first generation can be exchanged in the $t$-channel ( $F=0)$ or in the $u$-channel $(F=2)$. The coupling of leptoquarks to quark-lepton pairs, $g$, is referred to as $g_{\mathrm{L}}$ or $g_{\mathrm{R}}$, according to the chirality of the lepton. The contributions of leptoquark exchange to $\mathrm{e}^{+} \mathrm{e}^{-}$ $\rightarrow \mathrm{q} \overline{\mathrm{q}}$ depend on $g^{2}$.

Studying the exchange of different types of leptoquarks separately, limits on $|g|$ are derived depending on the mass, $m_{\mathrm{LQ}}$, of the exchanged leptoquark. The states $S_{0}, S_{1 / 2}$ and $V_{0}, V_{1 / 2}$ couple to both leftand right-handed quarks. Here, only $g_{\mathrm{L}}$ or $g_{\mathrm{R}}$ is assumed to be non-zero since low energy processes and rare decays of $\pi$ and $\mathrm{K}$ constrain the product $g_{\mathrm{L}} g_{\mathrm{R}}[38,39]$. Upper limits on the allowed values for $|g|$ are presented in Fig. 3 for scalar leptoquarks and in Fig. 4 for vector leptoquarks.

For a coupling of electromagnetic strength, $g$ $=\sqrt{4 \pi \alpha}$, where $\alpha$ is the fine-structure constant, mass limits can be derived. The results for these lower bounds on leptoquark masses are given in Table 4 . In case of $\tilde{S}_{1 / 2}(\mathrm{~L})$ exchange, i.e. coupling to left-handed fermions, the assumption $g_{\mathrm{L}}=\sqrt{4 \pi \alpha}$ yields a very small contribution to the hadron cross section that is not observable with the precision of our measurements.

The results at LEP complement the leptoquark searches at HERA. In most cases the indirect limits on leptoquark masses and couplings obtained in our analysis are more stringent than the corresponding limits presented by the $\mathrm{H} 1$ collaboration [34]. The indirect search covers regions at high leptoquark

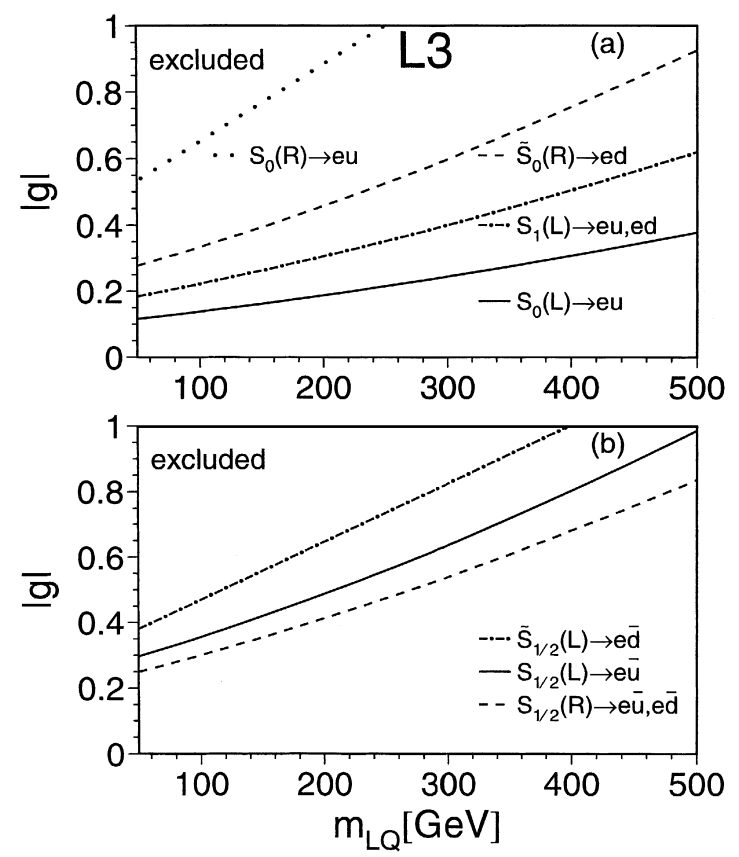

Fig. 3. The $95 \%$ confidence level upper limits on $\left|g_{L}\right|$ or $\left|g_{R}\right|$ as a function of $m_{\mathrm{LQ}}$ for various scalar leptoquarks derived from hadronic final state cross sections. Limits are shown for fermion number $F=2$ (a) and for $F=0$ (b). Bounds on the R-parity violating couplings $\left|\lambda_{1 j k}^{\prime}\right|$ for the exchange of scalar down-type quarks in the $u$-channel and scalar up-type quarks in the $t$-channel correspond to limits on $\left|g_{\mathrm{L}}\right|$ for the $S_{0}(\mathrm{~L})$ and $\tilde{S}_{1 / 2}$ leptoquark exchange, respectively. 

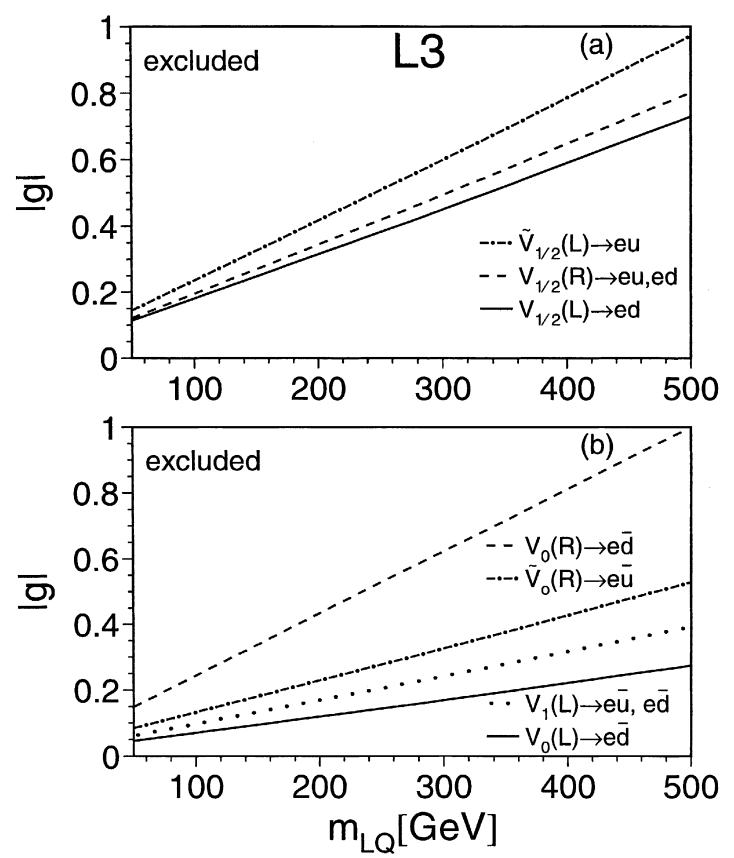

Fig. 4. The $95 \%$ confidence level upper limits on $\left|g_{L}\right|$ or $\left|g_{R}\right|$ as a function of $m_{\mathrm{LQ}}$ for various vector leptoquarks derived from hadronic cross sections. Limits are shown for fermion number $\mathrm{F}=2$ (a) and for $\mathrm{F}=0$ (b).

masses above the reach of direct leptoquark searches [40-43].

\section{R-parity violating scalar neutrinos and scalar quarks}

Even in a minimal supersymmetric model [44] the most general superpotential contains interactions violating R-parity in the trilinear couplings of super- fields. The only renormalisable gauge invariant operator that couples fermions and their scalar partners is given by $[45,46]$ :

$W_{R}=\lambda_{i j k} L_{i} L_{j} \bar{E}_{k}+\lambda_{i j k}^{\prime} L_{i} Q_{j} \bar{D}_{k}+\lambda_{i j k}^{\prime \prime} \bar{U}_{i} \bar{D}_{j} \bar{D}_{k}$,

where $L$ and $E$ are the leptonic, and $Q, U$ and $D$ are the quark superfields. The family indices are $i, j$ and $k$, e.g. $\lambda_{121}$ for $\tilde{v}_{\mu}$ exchange in the reaction $\mathrm{e}^{+} \mathrm{e}^{-} \rightarrow$ $\mathrm{e}^{+} \mathrm{e}^{-}$.

The exchange of scalar neutrinos can produce resonance peaks at LEP energies. From an analysis of our measurements in the leptonic channels upper limits on the coupling strength $\lambda$ as a function of the scalar neutrino mass are determined. The results for the $\mathrm{e}^{+} \mathrm{e}^{-}, \mu^{+} \mu^{-}$and $\tau^{+} \tau^{-}$final states are shown in Fig. 5. In all cases, large and previously unexplored areas in the $\left(m_{\tilde{v} \mu}, \lambda_{121}\right),\left(m_{\tilde{v} \tau}, \lambda_{131}\right),\left(m_{\tilde{\nu} \tau}, \sqrt{\lambda_{131} \lambda_{232}}\right)$ and $\left(m_{\tilde{\nu} \mu}, \sqrt{\lambda_{121} \lambda_{233}}\right)$ planes are excluded.

From the analysis of the hadronic cross section measurements, upper limits on the Yukawa couplings $\left|\lambda_{1 j k}^{\prime}\right|(j, k=1,2,3)$ are derived depending on the mass of exchanged scalar quarks. One single Yukawa coupling at a time is assumed to be much larger than the others which are neglected. Two cases are analysed:

$m_{\tilde{U}} \gg m_{\tilde{D}} \quad$ with $\tilde{U}=\tilde{\mathrm{u}}, \tilde{\mathrm{c}}, \tilde{\mathrm{t}}$ and $\tilde{D}=\tilde{\mathrm{d}}, \tilde{\mathrm{s}}, \tilde{\mathrm{b}}$

$m_{\tilde{U}} \ll m_{\tilde{D}}$.

Only the exchange of the much lighter scalar quark type is important. Due to quark universality the limits on $\left|\lambda_{1 j k}^{\prime}\right|$ coincide for each of the two cases of mass relation.

The R-parity breaking Yukawa couplings are mainly restricted by virtual exchange of right-handed

Table 4

Lower limits on the mass of leptoquarks at $95 \%$ confidence level derived from hadronic cross section measurements assuming $g=\sqrt{4 \pi \alpha}$

\begin{tabular}{lcll}
\hline LQ type & $m_{\mathrm{LQ}}[\mathrm{GeV}]$ & LQ type & $m_{\mathrm{LQ}}[\mathrm{GeV}]$ \\
\hline$S_{0}(\mathrm{~L}) \rightarrow \mathrm{eu}$ & 390 & $V_{1 / 2}(\mathrm{~L}) \rightarrow \mathrm{ed}$ & 190 \\
$S_{0}(\mathrm{R}) \rightarrow \mathrm{eu}$ & 300 & $V_{1 / 2}(\mathrm{R}) \rightarrow \mathrm{eu}, \mathrm{ed}$ & 170 \\
$\tilde{S}_{0}(\mathrm{R}) \rightarrow \mathrm{ed}$ & 80 & $\tilde{V}_{1 / 2}(\mathrm{~L}) \rightarrow \mathrm{eu}$ & 140 \\
$S_{1}(\mathrm{~L}) \rightarrow \mathrm{eu}, \mathrm{ed}$ & 200 & $V_{0}(\mathrm{~L}) \rightarrow \mathrm{e} \overline{\mathrm{d}}$ & 560 \\
$S_{1 / 2}(\mathrm{~L}) \rightarrow \mathrm{eu}$ & 55 & $V_{0}(\mathrm{R}) \rightarrow \mathrm{e} \overline{\mathrm{d}}$ & 130 \\
$S_{1 / 2}(\mathrm{R}) \rightarrow \mathrm{eu}, \mathrm{e} \overline{\mathrm{d}}$ & 110 & $\tilde{V}_{0}(\mathrm{R}) \rightarrow \mathrm{eu}$ & 280 \\
$\tilde{S}_{1 / 2}(\mathrm{~L}) \rightarrow \mathrm{e} \overline{\mathrm{d}}$ & - & $V_{1}(\mathrm{~L}) \rightarrow \mathrm{eu}, \mathrm{e} \overline{\mathrm{d}}$ & 380 \\
\hline
\end{tabular}



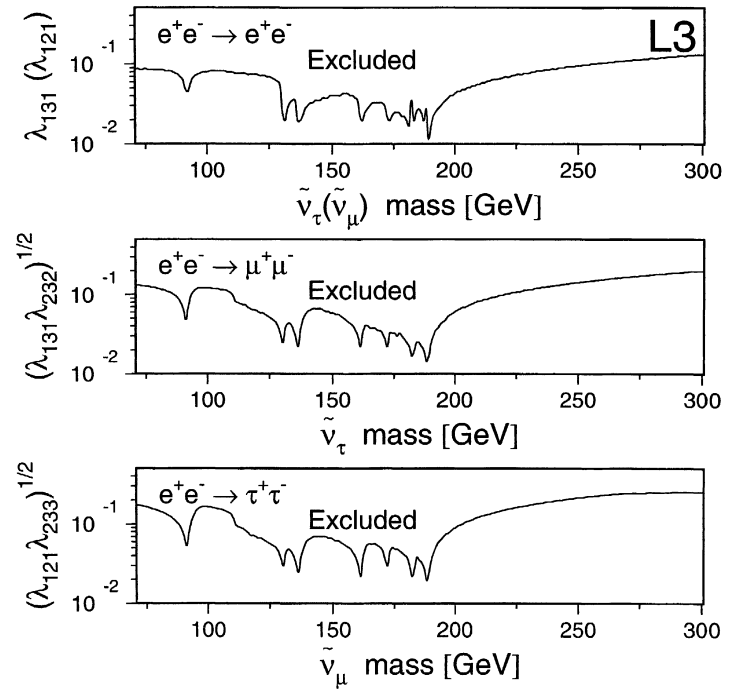

Fig. 5. Upper limits at $95 \%$ confidence level on the coupling strengths $\lambda_{i j k}$ of scalar leptons to leptons as a function of the scalar neutrino mass, derived from measurements of lepton-pair production $\mathrm{e}^{+} \mathrm{e}^{-}, \mu^{+} \mu^{-}$and $\tau^{+} \tau^{-}$.

scalar down-type quarks in the $u$-channel which couple in the same way as $S_{0}$ leptoquarks with $\left|g_{\mathrm{L}}\right|$. Their amplitudes interfere with the equal-helicity amplitude (LL) of the Standard Model.

The amplitudes for left-handed scalar up-type quark exchange in the $t$-channel are the same as for $\tilde{S}_{1 / 2}$ leptoquark exchange and interfere with the opposite-helicity amplitude (LR). The latter is suppressed in comparison to (LL). The results on $\left|\lambda_{1 j k}^{\prime}\right|$ can be taken from Fig. 3 considering $S_{0}(\mathrm{~L})$ and $\tilde{S}_{1 / 2}$. Assuming scalar up-type and down-type quark masses to be equal and both contributing to the hadronic cross section yields similar limits as for the case $m_{\tilde{U}} \gg m_{\tilde{D}}$.

\section{TeV strings}

Recently, it has been realized that in a string theory of quantum gravity $[9,10]$ there are new phenomenological consequences. For instance, massive string mode oscillations can lead to contact interactions, which may have stronger effects than those caused by the virtual exchange of gravitons.
The effects of $\mathrm{TeV}$ scale strings on Bhabha scattering are computed [10] by multiplying the leadingorder scattering amplitudes by a common form factor, which depends on the string scale $M_{S}$ and the Mandelstam variables $s$ and $t$. The Standard Model cross section for Bhabha scattering is modified as follows:

$$
\frac{d \sigma}{d \cos \theta}=\left(\frac{d \sigma}{d \cos \theta}\right)_{S M}\left|\frac{\Gamma\left(1-\frac{s}{M_{S}^{2}}\right) \Gamma\left(1-\frac{t}{M_{S}^{2}}\right)}{\Gamma\left(1-\frac{s}{M_{S}^{2}}-\frac{t}{M_{S}^{2}}\right)}\right|^{2}
$$

where $\Gamma$ is the gamma function.

The differential cross sections measured at 183 and $189 \mathrm{GeV}$ are used to derive a lower limit on the string scale $M_{S}$ of $0.49 \mathrm{TeV}$. The result of the analysis at $189 \mathrm{GeV}$ is depicted in Fig. 6 .

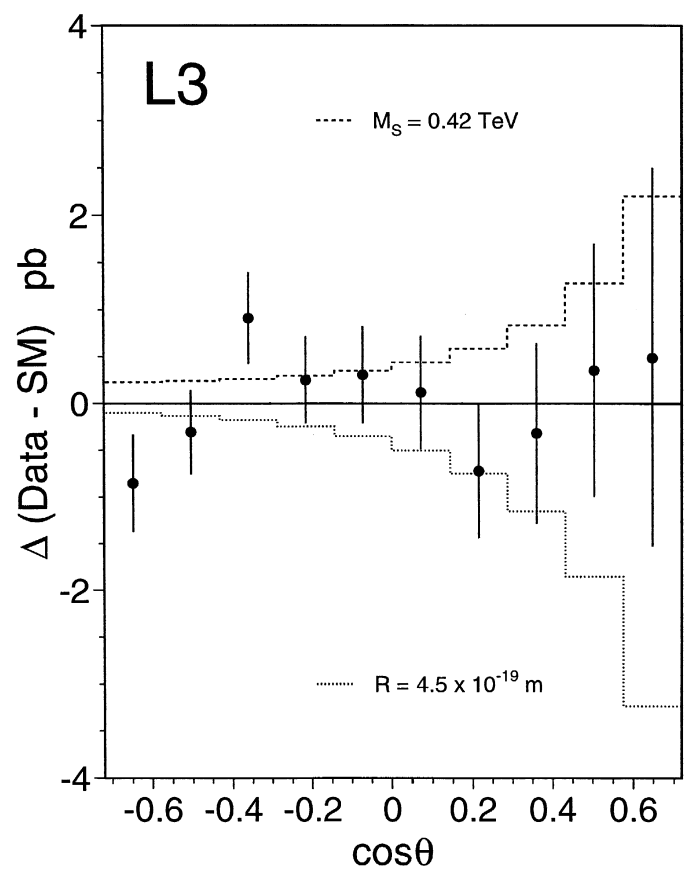

Fig. 6. Deviations of the measured differential cross section for Bhabha scattering at $\sqrt{s}=189 \mathrm{GeV}$ from the Standard Model prediction. The effects expected in string models and for non-zero electron size are also shown. 


\section{Form factors and fermion sizes}

In the Standard Model the fermions and the gauge bosons are considered to be pointlike. If this is not the case, form factors or anomalous magnetic dipole moments of the fermions could be observed [47].

The fermion-pair measurements above the $\mathrm{Z}$ pole are analysed for such effects. The Standard Model cross sections for the reactions $\mathrm{e}^{+} \mathrm{e}^{-} \rightarrow \overline{f f}$ are modified as follows:

$$
\frac{d \sigma}{d q^{2}}=\left(\frac{d \sigma}{d q^{2}}\right)_{S M} F_{\mathrm{e}}^{2}\left(q^{2}\right) F_{f}^{2}\left(q^{2}\right),
$$

where $q^{2}$ is the Mandelstam variable $s$ or $t$ for $s$ - or $t$-channel exchange, and the form factors of the initial and final state fermions are denoted as $F_{e}$ and $F_{f}$, respectively. They are parametrized by a Dirac form factor:

$F\left(q^{2}\right)=1+\frac{1}{6} q^{2} R^{2}$,

where $R$ is the radius of the fermion.

The upper limits on the fermion radii obtained from our data are shown in Table 5. They are derived with the assumption $F_{e}=F_{f}$. For the $\mu^{+} \mu^{-}$, $\tau^{+} \tau^{-}$and $\mathrm{q} \overline{\mathrm{q}}$ final states the limits given in Table 5 will increase by a factor of $\sqrt{2}$ under the most conservative assumption that the electron is pointlike $\left(F_{e} \equiv 1\right)$. The expected effects on the differential cross section for the $\mathrm{e}^{+} \mathrm{e}^{-}$final state are shown in Fig. 6.

The limits for quarks derived in this paper are more stringent than similar limits from high energy analyses of interactions involving quarks and elec-

Table 5

Upper limits on the fermion radii at $95 \%$ confidence level for electrons, muons, taus, all leptons combined, and for quarks

\begin{tabular}{ll}
\hline Channel & $R[\mathrm{~m}]$ \\
\hline $\mathrm{e}^{+} \mathrm{e}^{-}$ & $3.1 \cdot 10^{-19}$ \\
$\mu^{+} \mu^{-}$ & $2.4 \cdot 10^{-19}$ \\
$\tau^{+} \tau^{-}$ & $4.0 \cdot 10^{-19}$ \\
$\ell^{+} \ell^{-}$ & $2.2 \cdot 10^{-19}$ \\
$\mathrm{q} \overline{\mathrm{q}}$ & $3.0 \cdot 10^{-19}$ \\
\hline
\end{tabular}

trons by the $\mathrm{H} 1$ collaboration [34] in deep inelastic scattering, and by the CDF collaboration [35] from study of the Drell-Yan process.

Limits on lepton radii have been extracted from the high precision low energy measurements of the magnetic dipole moment $(g-2)$ of the electron and the muon $[47,48]$. In the case where the deviations from the Standard Model of the magnetic dipole moments of the leptons depend linearly on their mass, the measurements of dipole moments can be interpreted as giving much more stringent limits. By contrast, in the case where the deviations depend quadratically on the masses, our limit on the electron size is one order of magnitude lower, and our limit on the muon size is similar to the limits derived from $(g-2)$ measurements.

\section{Conclusions}

The measurements of fermion-pair cross sections and forward-backward asymmetries, performed with the L3 detector at centre-of-mass energies between $130 \mathrm{GeV}$ and $189 \mathrm{GeV}$, are used to search for effects of new physics phenomena. No hint of manifestations of physics beyond the Standard Model is found.

The sensitivity of the searches, performed at energies above the $\mathrm{Z}$ pole, has improved substantially compared to our previous publications. Limits on the energy scale $\Lambda$ of four-fermion contact interactions in the range 3.8-14.4 TeV for leptons, and in the range 2.8-6.1 TeV for quarks are obtained. The effects of the exchange of leptoquarks or R-parity violating scalar quarks and scalar neutrinos are studied. In both cases, upper limits on the coupling constants, $\left|g_{\mathrm{L}}\right|$ and $\left|g_{\mathrm{R}}\right|$, or $\left|\lambda^{\prime}\right|$ and $|\lambda|$ are determined as a function of the particle masses. Lower limits on the mass of leptoquarks between $55 \mathrm{GeV}$ and $560 \mathrm{GeV}$, depending on the leptoquark type, are derived assuming $g=\sqrt{4 \pi \alpha}$.

In addition, new searches are performed for the effects of $\mathrm{TeV}$ strings, predicted in quantum gravity models, and a lower limit on the string scale $M_{S}$ of $0.49 \mathrm{TeV}$ is set. From an analysis of form factors, upper limits on the size of the different leptons and quarks in the range $(2.2-4.0) 10^{-19} \mathrm{~m}$ are derived. 


\section{Acknowledgements}

We are grateful to M. Peskin for stimulating discussions. We wish to express our gratitude to the CERN accelerator divisions for the excellent performance of the LEP machine. We acknowledge the contributions of the engineers and technicians who have participated in the construction and maintenance of this experiment.

\section{References}

[1] L3 Collaboration, M. Acciarri et al., Phys. Lett. B 414 (1997) 373.

[2] L3 Collaboration, M. Acciarri et al., Phys. Lett. B 433 (1998) 163.

[3] L3 Collaboration, M. Acciarri et al., Phys. Lett. B 370 (1996) 195.

[4] L3 Collaboration, M. Acciarri et al., Phys. Lett. B 407 (1997) 361.

[5] L3 Collaboration, M. Acciarri et al., preprint CERN-EP/99181, hep-ex /0002034, accepted by Phys. Lett. B.

[6] ALEPH Collaboration, R. Barate et al., Eur. Phys. J. C 12 (2000) 183.

[7] DELPHI Collaboration, P. Abreu et al., Eur. Phys. J. C 11 (1999) 383.

[8] OPAL Collaboration, G. Abbiendi et al., preprint CERNEP /99-097, accepted by Eur. Phys. J.C.

[9] E. Accomando, I. Antoniadis, K. Benakli, hep-ph/9912287.

[10] S. Cullen, M. Perelstein, M. Peskin, hep-ph/0001166.

[11] N. Arkani-Hamed, S. Dimopoulos, G. Dvali, Phys. Lett. B 429 (1998) 263.

[12] I. Antoniadis et al., Phys. Lett. B 436 (1998) 257.

[13] N. Arkani-Hamed, S. Dimopoulos, G. Dvali, Phys. Rev. D 59 (1999) 086004.

[14] L3 Collaboration, M. Acciarri et al., Phys. Lett. B 464 (1999) 135.

[15] L3 Collaboration, M. Acciarri et al., Phys. Lett. B 470 (1999) 281.

[16] D. Bourilkov, J. High Energy Phys. 08 (1999) 006.

[17] D. Bourilkov, hep-ph/0002172.

[18] L3 Collaboration, B. Adeva et al., Nucl. Instr. Meth. A 289 (1990) 35.

[19] M. Acciarri et al., Nucl. Instr. Meth. A 351 (1994) 300.

[20] M. Chemarin et al., Nucl. Instr. Meth. A 349 (1994) 345.

[21] I.C. Brock et al., Nucl. Instr. Meth. A 381 (1996) 236.

[22] A. Adam et al., Nucl. Instr. Meth. A 383 (1996) 342.

[23] ZFITTER version 6.21 is used. D. Bardin et al., hepph/9908433; Z. Phys. C 44 (1989) 493; Nucl. Phys. B 351 (1991) 1; Phys. Lett. B 255 (1991) 290. For the comparison with our measurements, the following ZFITTER flags have been changed from their default values: $\mathrm{FINR}=0, \mathrm{INTF}=0$, and $\mathrm{BOXD}=2$.

[24] S.L. Glashow, Nucl. Phys. 22 (1961) 579; S. Weinberg, Phys. Rev. Lett. 19 (1967) 1264; A. Salam, Elementary Particle Theory, N. Svartholm (Ed.), Stockholm, Almquist \& Wiksell, 1968, p. 367.

[25] TOPAZ0 version 4.4 is used. G. Montagna et al., Nucl. Phys. B 401 (1993) 3; Comp. Phys. Comm. 76 (1993).

[26] L3 Collaboration, M. Acciarri et al., Z. Phys. C 62 (1994) 551.

[27] L3 Collaboration, O. Adriani et al., Phys. Rep. 236 (1993) 1.

[28] S. Eidelmann, F. Jegerlehner, Z. Phys. C 67 (1995) 585.

[29] Particle Data Group, C. Caso et al., Eur. Phys. J. C 3 (1998) 1.

[30] CDF Collaboration, F. Abe et al., Phys. Rev. Lett. 82 (1999) 2808; DØCollaboration, S. Abachi et al., Phys. Rev. Lett. 79 (1997) 1197; we use the average top mass as given in Ref. [29]

[31] E. Eichten, K. Lane, M. Peskin, Phys. Rev. Lett. 50 (1983) 811.

[32] C.S. Wood et al., Science 275 (1997) 1759; V. Barger et al., Phys. Lett. B 404 (1997) 147; N. Di Bartolomeo, M. Fabbrichinesi, Phys. Lett. B 406 (1997) 237.

[33] ZEUS Collaboration, J. Breitweg et al., E. Phys. J. C(2000) http://dx.doi.org/10.1007/s100520000336.

[34] H1 Collaboration, C. Adloff et al., preprint DESY 00-027, accepted by Phys. Lett. B.

[35] CDF Collaboration, F. Abe et al., Phys. Rev. Lett. 79 (1997) 2198.

[36] DØCollaboration, B. Abbott et al., Phys. Rev. Lett. 82 (1999) 4769.

[37] A. Djouadi et al., Z. Phys. C 46 (1990) 679; B. Schrempp, in: W. Buchmüller, G. Ingelman (Eds.). Proc., Physics at HERA, Hamburg 1991.

[38] M. Leurer, Phys. Rev. D 49 (1994) 333; Phys. Rev. D 50 (1994) 536.

[39] S. Davidson, D. Bailey, D. Campbell, Z. Phys. C 61 (1994) 613; M. Hirsch, H.V. Klapdor-Kleingrothaus, S.G. Kovalenko, Phys. Rev. D 54 (1996) R4207.

[40] H1 Collaboration, C. Adloff et al., Eur. Phys. J. C 11 (1999) 447.

[41] ZEUS Collaboration, J. Breitweg et al., preprint DESY 00023, accepted by Eur. Phys. J.C.

[42] CDF Collaboration, F. Abe et al., Phys. Rev. Lett. 79 (1997) 4327.

[43] DØCollaboration, B. Abbott et al., Phys. Rev. Lett. 80 (1998) 2051.

[44] Y.A. Golfand, E.P. Likhtman, Sov. Phys. JETP 13 (1971) 323; D.V. Volkhov, V.P. Akulov, Phys. Lett. B 46 (1973) 109; J. Wess, B. Zumino, Nucl. Phys. B 70 (1974) 39; P. Fayet, S. Ferrara, Phys. Rep. 32 (1977) 249; A. Salam, J. Strathdee, Fortschr. Phys. 26 (1978) 57.

[45] S. Dimopoulos, L. Hall, Phys. Lett. B 207 (1987) 210.

[46] V. Barger, G. Giudice, T. Han, Phys. Rev. D 40 (1989) 2987.

[47] G. Köpp et al., Z. Phys. C 65 (1995) 545.

[48] S.J. Brodsky, S.D. Drell, Phys. Rev. D 22 (1980) 2236. 